\title{
Acute thrombosis in superior mesenteric artery as first symptom in a AML patient
}

\author{
Yan Liu' \\ Xangshan Chao' \\ Weiying $\mathrm{Gu}^{\prime}$ \\ Xiaoying Hua' \\ Ning $X u^{2}$ \\ 'Department of Hematology, The \\ Third Affiliated Hospital, Suzhou \\ University, Changzhou, China; \\ ${ }^{2}$ Division of Clinical Chemistry \\ and Pharmacology, Department of \\ Laboratory Medicine, Lund University, \\ Lund, Sweden
}

Correspondence: Ning Xu

Division of Clinical Chemistry and

Pharmacology, Department of Laboratory

Medicine, Lund University, S-22I 85 Lund,

Sweden

Tel $+4646 \quad 173462$

Fax $+4646 \quad 130064$

Email ning.xu@med,lu.se

\begin{abstract}
It is well known that acute leukemia may accompany thromboembolic events; even severe thrombocytopenia does not prevent thrombosis. Coagulation dysfunction is the major pathophysiological background for thromboembolism in these patients. Most thromboembolism is localized in venous vessels in acute leukemic patients and it happens rarely in the artery. We report a case of acute thrombosis in the superior mesenteric artery as the first symptom in a patient suffering from acute myeloid leukemia (FAB M4).
\end{abstract}

Keywords: acute leukemia, thromboembolism, pathogenesis

\section{Case report}

A 56-year-old man suffered from a sudden onset of severe pain in upper abdomen together with vomiting for three hours. The pain was persistent without intermission. The patient was urgently admitted to our hospital on January 31, 2007. Abdominal CT revealed that there was a thromboembolism in superior mesenteric artery. The patient was healthy without any cardiovascular or metabolic disease previously, and had neither personal nor familial thromboembolic history. Body examination showed a normal status except weakened bowel sounds. Laboratory examinations showed that hemoglobin was $102 \mathrm{~g} / 1$, white blood cell (WBC), $5.77 \times 10^{9} / 1$ and platelet, $201 \times 10^{9} / 1$. Lactate dehydrogenase (LDH) level increased (496 U/l). Prothrombin time (PT), active partial thromboplastin time (APTT), thrombin time (TT), and plasma fibrinogen level were at normal ranges. Emergent surgical embolectomy was performed successfully, which revealed a hard thrombus in the superior mesenteric artery. After surgery the patient received standard treatment of heparinization and AT-III substitution. On the third day post-operation, the patient had symptoms of high fever, fatigue, and weakness. Further blood examination showed that hemoglobin was only $78 \mathrm{~g} / \mathrm{l}$, WBC $20 \times 10^{9} / 1$ and platelets $78 \times 10^{9} / 1$, and a few blast cells were identified on the peripheral smear. A subsequent bone marrow examination confirmed diagnosis of an acute myeloid leukemia (AML) FAB M4. Myeloblasts was 60\%, monocytic cells was $22 \%$, and there was no abnormal eosiniphilia. Chromosomal analyses showed a normal karyotype $46, \mathrm{XX}$ without any abnormalities, and there was no translocation of $t(15 ; 17)(\mathrm{q} 22 ; \mathrm{q} 21), t(11 ; 17)(\mathrm{q} 23 ; \mathrm{q} 21)$, inv(16)(p13; q22) or t(16;16) (p13; q22) in blast cells. The polymerase chain reaction for the BCR/ABL, PML/RARa, AML1-ETO, or MLL rearrangement was negative. Immunophenotypical characterization of bone marrow cells by the flow-cytometry showed AML with expression of CD13, CD14, and CD33. A standard induction chemotherapy protocol (Idarubicin and cytarabine) did not get success of complete remission. The patient had complete remission after second chemotherapy (fludarabine $50 \mathrm{mg} / \mathrm{d} \times 5 \mathrm{~d}$, cytarabine $2.0 \mathrm{~g} / \mathrm{d} \times 6 \mathrm{~d}$ and G-CSF $300 \mu \mathrm{g} / \mathrm{d}$ ). After two courses of consolidation treatment: one was fludarabine $50 \mathrm{mg} / \mathrm{d} \times 3 \mathrm{~d}$, cytarabine $1.0 \mathrm{~g} / \mathrm{d} \times 5 \mathrm{~d}$ and G-CSF $300 \mu \mathrm{g} / \mathrm{d}$, another was mitoxantrone 
$5 \mathrm{mg} / \mathrm{d} \times 3 \mathrm{~d}$, cytarabine $0.1 \mathrm{q} 12 \mathrm{~h} \times 7 \mathrm{~d}$ and etoposide $0.1 / \mathrm{d} \times 3 \mathrm{~d}$, the patient received an autoperipheral blood stem cell transplantation. After transplantation the patient presented with heart failure and renal failure. The hematopoiesis in his bone marrow did not completely recover though there was no blast in it. He died eight months after transplantation because of heart failure and renal failure.

\section{Discussion}

Thromboembolism is a common complication in the patients suffering from acute leukemia, especially in the acute promyelocytic leukemia (APL) patients. It occurs mostly in the venous system. In a fellow-up observational cohort study of 379 adult acute leukemic patients, De Stefano and colleagues (2005) demonstrated that there were 6.3\% of patients with thrombosis, $80 \%$ with venous thrombosis, and $20 \%$ with arterial. And distribution of cases were: $1.4 \%$ in ALL, 9.6\% in APL, and 3.2\% in non-M3 AML patients (De Stefano et al 2005). The pathogenesis of thrombosis in acute leukemic patients is complicated. Traditional view is based on the Virchow's triad, ie, slowed blood flow, impaired vessel integrity and alteration of blood components (Virchow 1998). New observations suggest that even more prothrombotic factors may potentiate the thromboembolic events in leukemic patients (Kwaan and Vicuna 2007): 1, Leukemic cells can produce several factors that may interfere coagulation and fibrinolytic systems, resulting in the hypercoagulable status in blood (Gale and Gordon 2001; Falanga 2005). 2, Apoptosis of leukemic cells occurs spontaneously during rapid cell proliferation or under chemotherapy, resulting in an increased prothrombotic risk. 3, Coagulation inhibitors, such as antithrombin and protein $\mathrm{C}$ system, may be inhibited in acute leukemic patients, which reduces the anticoagulation functions (Dixit et al 2006). 4, Certain inflammatory cytokines, such as interleukin (IL)-1 $\alpha$, IL-6, tissue necrosis factor-beta (TNF- $\beta$ ) could be produced by either leukemic cells or normal host cells, which might stimulate TF expression on monocytes and could also downregulate anticoagulant factors such as thrombomodulin, which may enhance procoagulant activities and further initiates the thrombosis (Rickles and Falanga 2001). Breccia and colleagues (2007) observed a close correlation between certain biological features of leukemic cells and the development of thrombotic events in APL patients. These characteristics included CD2 and/or CD15 expression, bcr3 PML/RARa transcript type, FLT3-ITD, and elevated WBC (Breccia et al 2007).

The incidence of symptomatic thrombosis in the early stage of ALL patients is relatively low (1.4\%), whereas it is significantly increased during the further chemotherapy, which may be up to $10.6 \%$ (De Stefano et al 2005). $\mathrm{L}$-asparaginase is an essential drug for ALL but its major complication is thrombosis. It might impair the balance of hemostatic system by reducing the synthesis of both coagulation factors and inhibitors. It is demonstrated that coagulation inhibitors, ie, antithrombin, protein $\mathrm{C}$ and proteins $\mathrm{S}$ were significantly reduced during asparaginase therapy (Ruud et al 2006). Thrombosis in central nervous system is a asparaginase-related thrombosis, which occurs in 1\%-3\% of patients (Payne and Vora 2007). Most of the thrombotic events occurred during the induction phase of therapy. Lower doses of asparaginase with a long period may associate with the highest incidence of thrombosis in ALL patients (Caruso et al 2006).

Although acute leukemic patients have high risk of thromboembolism, the preventive treatment of this complication is still controversial due to the higher risk of hemorrhage in the patients. Up to now, there is no recommended guideline of anticoagulant therapy in the acute leukemia patients except APL patients with high leukocyte counts or at high risk of developing ATRA syndrome (Chong and Lee 2007). Unfractionated heparin (UFH) and low molecular weight heparin (LMWH) are efficacious for the thromboembolism prophylaxis and treatment in acute leukemia patients. When the platelet count decreased below $50 \times 10^{9} / 1$ the risk of bleeding may significantly increased with anticoagulant prophylaxis (Tapson et al 2007).

\section{Conclusion}

The present case report indicates that an unexpected thromboembolic event in a healthy patient might also be the symptom of an underlying hematological disorder such as acute leukemia. Thromboembolism is a common complication in the patients suffering from acute leukemia, however preventive anticoagulant treatment should be considered according to patient's coagulation - anticoagulation balance.

\section{Disclosure}

The authors report no conflicts of interest in this work.

\section{References}

Breccia M, Avvisati G, Latagliata R, et al. 2007. Occurrence of thrombotic events in acute promyelocytic leukemia correlates with consistent immunophenotypic and molecular features. Leukemia, 21:79-83.

Caruso V, Iacoviello L, Di Castelnuovo A, et al. 2006. Thrombotic complications in childhood acute lymphoblastic leukemia: a meta-analysis of 17 prospective studies comprising 1752 pediatric patients. Blood, 108:2216-22.

Chong BH, Lee SH. 2007. Management of thromboembolism in hematologic malignancies. Semin Thromb Hemost, 33:435-48. 
De Stefano V, Sora F, Rossi E, et al. 2005. The risk of thrombosis in patients with acute leukemia: occurrence of thrombosis at diagnosis and during treatment. J Thromb Haemost, 3:1985-92.

Dixit A, Kannan M, Mahapatra M, et al. 2006. Roles of protein C, protein S, and antithrombin III in acute leukemia. Am J Hematol, 81:171-4.

Falanga A. 2005. Thrombophilia in cancer. Semin Thromb Hemost, 31:104-10.

Gale AJ, Gordon SG. 2001. Update on tumor cell procoagulant factors. Acta Haematologica, 106:25-32.

Kwaan HC, Vicuna B. 2007. Incidence and pathogenesis of thrombosis in hematologic malignancies. Semin Thromb Hemost, 33:303-12.

Payne JH, Vora AJ. 2007. Thrombosis and acute lymphoblastic leukaemia. Br J Haematol, 138:430-45.
Rickles FR, Falanga A. 2001. Molecular basis for the relationship between thrombosis and cancer. Thromb Res, 102:V21-5-24.

Ruud E, Holmstrom H, de Lange C, et al. 2006. Thrombotic effects of asparaginase in two acute lymphoblastic leukemia protocols (NOPHO ALL-1992 versus NOPHO ALL-2000): a single-institution study. Pediatr Hematol Oncol, 23:207-16.

Tapson VF, Decousus H, Pini M, et al. 2007. Venous thromboembolism prophylaxis in acutely ill hospitalized medical patients: findings from the International Medical Prevention Registry on Venous Thromboembolism. Chest, 132:936-45.

Virchow R. 1998. [Thrombosis and Emboli]. Translated by Matzdorff AC, Bell WR. Gesammelte Abhandlungen Zur Wissenschaftlichen Medicin, 1856:220-380. 
